\title{
Validación de Belief Medicines Questionnaire y Self-efficacy for Appropriate Medication Use Scale para medir adherencia al tratamiento farmacológico en pacientes con enfermedad inflamatoria intestinal
}

\author{
Katya Estefanía Bozada-Gutiérrez, ${ }^{1}$ Ana Fresán-Orellana² y Jesús K. Yamamoto-Furusho' \\ 'Secretaría de Salud, Instituto Nacional de Ciencias Médicas y Nutrición "Salvador Zubirán", Clínica de Enfermedad Inflamatoria Intestinal; \\ ${ }^{2}$ Secretaría de Salud, Instituto Nacional de Psiquiatría "Ramón de la Fuente Muñiz", Subdirección de Investigaciones Clínicas. Ciudad de México, \\ México
}

\section{Resumen}

Introducción: La adherencia terapéutica es crucial en la enfermedad inflamatoria intestinal (EII) para evitar recaídas y complicaciones. En México no se dispone de una herramienta validada para evaluar adherencia en pacientes con Ell. Objetivo: Traducir los instrumentos Belief Medicines Questionnaire (BMQ) y Self-Efficacy for Appropriate Medication Use Scale (SEAMS), y determinar su validez, fiabilidad y sensibilidad en pacientes mexicanos con diagnóstico de Ell. Método: Se incluyeron 149 pacientes con diagnóstico de Ell, previo consentimiento informado. Se tradujeron los instrumentos al español y posteriormente fueron aplicados durante la consulta médica. Para la SEAMS se realizó análisis factorial exploratorio, análisis de curva ROC y determinación del alpha de Cronbach; para el BMQ se empleó el coeficiente kappa de Cohen y su capacidad predictiva. Resultados: Se incluyeron 75 mujeres (50.3\%) con edad promedio de 44 años. La escala SEAMS mostró un único factor altamente confiable (alfa de Cronbach $=0.92$ ) y un punto de corte de 33 para identificar a los pacientes adherentes. Las dimensiones "adherencia" y "barrera de recuerdo" del BMQ fueron adecuados predictores de adherencia. Conclusiones: Las versiones en español SEAMS y BMQ son válidas para medir autoeficacia y barreras para la adherencia al tratamiento farmacológico en pacientes mexicanos con Ell.

PALABRAS CLAVE: Adherencia. Autoeficacia. Barreras. Tratamiento farmacológico. Enfermedad inflamatoria intestinal.

\begin{abstract}
Introduction: Treatment adherence is crucial in inflammatory bowel disease (IBD) to prevent relapses and complications. In Mexico, there is not a validated tool to assess adherence in patients with IBD. Objective: To translate the beliefs about medicines questionnaire (BMQ) and self-efficacy for appropriate medication use scale (SEAMS) instruments, as well as to determine their validity, reliability and sensitivity in IBD-diagnosed Mexican patients. Method: After informed consent was obtained, 149 IBD-diagnosed patients were included. The instruments were translated into Spanish and were subsequently applied during medical consultation. For SEAMS, exploratory factorial analysis and ROC curve analysis were carried out and Cronbach's alpha was determined; for the BMQ, Cohen's kappa coefficient and its predictive capacity were employed. Results: Seventy-five women (50.3\%) were included, with an average age of 44 years. The SEAMS scale showed a single factor that was highly reliable (Cronbach's alpha $=0.92$ ) and a cutoff point of 33 to identify adherent patients. The "adherence" and "recall barrier" dimensions of the BMQ were adequate adherence predictors. Conclusions: The SEAMS and BMQ Spanish versions are valid for measuring self-efficacy and barriers to pharmacological treatment adherence in Mexican patients with IBD.
\end{abstract}

KEY WORDS: Adherence. Self-efficacy. Barriers. Pharmacological treatment. Inflammatory bowel disease.

Correspondencia:

Jesús K. Yamamoto-Furusho

E-mail: kazuofurusho@hotmail.com
Fecha de recepción: 23-05-2018

Fecha de aceptación: 16-11-2018

DOI:10.24875/GMM.18004417
Gac Med Mex. 2019;155:124-129

Disponible en PubMed

www.gacetamedicademexico.com 


\section{Introducción}

La enfermedad inflamatoria intestinal (EII) incluye la colitis ulcerosa crónica idiopática (CUCl) y la enfermedad de Crohn, ${ }^{1}$ padecimientos inflamatorios crónicos en los que el tratamiento médico es indispensable de por vida, por lo que la adherencia al tratamiento farmacológico (ATF) resulta de suma importancia. La Organización Mundial de la Salud² define a la adherencia como: "grado de apego de una persona para la toma de medicamentos, seguimiento de un régimen alimentario y ejecutar cambios del estilo de vida de acuerdo con las recomendaciones acordadas con un prestador de asistencia sanitaria".

A pesar de la disponibilidad de numerosos tratamientos farmacológicos efectivos para el manejo de la Ell, la falta de adherencia por parte de los pacientes a la prescripción de medicamentos representa el mayor obstáculo en la efectividad terapéutica y provoca resultados negativos en los pacientes que derivan en aumento de la actividad de la enfermedad o reducción de la eficacia del tratamiento, ${ }^{3}$ aumento del riesgo de recaídas, ${ }^{4}$ mayor riesgo de hospitalización y prolongación de la estancia, ${ }^{5}$ que a su vez ocasionan mayor uso de los recursos sanitarios y peor calidad de vida. ${ }^{6}$

Diversas investigaciones han evaluado el papel de la ATF en los pacientes con Ell. En una reciente revisión sistemática se concluyó que la falta de adherencia a los medicamentos con 5-aminosalicitalos incrementa el riesgo de recaída en pacientes con CUCl. ${ }^{7}$ Además, el curso continuado de 5-aminosalicilatos puede reducir el riesgo de desarrollar cáncer colorrectal en este grupo de pacientes. ${ }^{8}$ En un estudio retrospectivo de 10 años en Estados Unidos que incluyó 13062 pacientes con $\mathrm{CUCl}$, con una mediana de seguimiento de 6.1 años, se observó que en los pacientes con baja adherencia a la mesalazina (5-aminosalicilato) oral aumentó significativamente el riesgo de brotes de $\mathrm{CUCl}$ en comparación con los adherentes altos $(R R=2.8, p<0.001) .{ }^{9}$

Existen dos tipos de métodos para medir la adherencia al tratamiento: directa e indirectamente ${ }^{10} \mathrm{sin}$ embargo, ninguno ha sido considerado por sí solo como estándar de oro. Los métodos indirectos, principalmente los cuestionarios autoaplicables, cuentan con la ventaja de ser sencillos, prácticos y reproducibles en la práctica médica; constituyen la mejor manera de aproximar al médico a la ATF de los pacientes. En una revisión sistemática, Nguyen et al.11 identificaron 43 escalas validadas para medir ATF, de las cuales las más utilizadas son la escala Morisky-Green, ${ }^{12}$ MARS,$^{13}$ The Beliefs about Medicines Questionnaire (BMQ) ${ }^{14}$ y SEAMS. ${ }^{15}$

La escala SEAMS, ${ }^{15}$ diseñada por Bandura et al., ${ }^{16}$ mide la autoeficacia, cuya base es la convicción, certeza o confianza del paciente para efectuar exitosamente una determinada conducta y producir un determinado resultado: ${ }^{17}$ la ATF. Por otro lado, $\mathrm{BMQ}^{14}$ se enfoca en identificar preocupaciones, creencias y barreras negativas acerca del tratamiento, las cuales influyen en la ATF, la cual ha sido validada en diversas patologías: asma,$^{18}$ infección por el virus de la inmunodeficiencia humana, ${ }^{19}$ trastornos psiquiatricos, ${ }^{20}$ artritis reumatoide, ${ }^{21}$ Ell. ${ }^{22}$

Reconociendo el impacto negativo de la falta de ATF en el curso clínico de la Ell, surgió la necesidad de contar con instrumentos validados que ayuden a los gastroenterólogos a evaluarla.

El presente estudio tiene como objetivo traducir y validar las escalas SEAMS y BMQ para medir adherencia, autoeficacia y barreras al tratamiento farmacológico en pacientes mexicanos con Ell.

\section{Método}

El presente estudio fue aprobado por los comités de investigación y ética del Instituto Nacional de Ciencias Médicas y Nutrición "Salvador Zubirán", donde se realizó, de acuerdo con las normas de las buenas prácticas clínicas.

Del 1 de agosto de 2017 al 31 de enero de 2018 se incluyeron pacientes entre 18 y 70 años de la Clínica de Ell del Instituto, que tuvieran el diagnóstico de Ell confirmado por histopatología y se encontraran en seguimiento por más de seis meses, bajo tratamiento farmacológico al momento del estudio, que supieran leer y escribir. Fueron excluidos quienes no estuvieran tomando tratamiento farmacológico o entregaran incompletos los instrumentos.

Con previo permiso por parte de los autores originales de SEAMS y BMQ, se tradujeron y adaptaron los instrumentos al español de acuerdo con la metodología de traducción directa e inversa recomendada. ${ }^{23}$ El reclutamiento de los pacientes comenzó después de terminar el proceso de traducción.

\section{Instrumentos de evaluación y procedimiento}

- SEAMS: $:^{15}$ instrumento autoaplicable de 13 reactivos que mide la autoeficacia de los pacientes 
respecto a la toma de los medicamentos. Los pacientes indican, de acuerdo con 13 situaciones preguntadas en los reactivos, la seguridad de tomar sus medicamentos correctamente: 1 , no seguro; 2, algo seguro; 3, muy seguro. Los resultados de la sumatoria van en un rango de 13-39 puntos, donde puntuaciones más altas indican óptima autoeficacia para la toma del tratamiento farmacológico.

- BMQ:14 escala estandarizada de 17 reactivos que evalúa las preocupaciones específicas sobre la medicación que la persona está tomando y las creencias sobre su importancia. La versión original está compuesta por dos secciones, BMQ-general (siete reactivos) y BMQ-específico (10 reactivos). Los reactivos se evalúan mediante una escala de cinco puntos que van de 1 , totalmente en desacuerdo, a 5, totalmente de acuerdo. El BMQ-general incluye las subescalas abuso y daño y El BMQ-específico las subescalas necesidad percibida de la medicación y preocupación sobre las consecuencias de la medicación en relación con el tratamiento farmacológico de la Ell. Al final, la sumatoria de las cuatro dimensiones genera una puntuación entre 0 a 4 puntos, donde 0 es adherencia al tratamiento y no existencia de barreras para la adherencia y 4 , no adherencia y presencia de alguna de las tres barreras para la adherencia (creencias, olvido y acceso al medicamento).

- Escala Visual Análoga (EVA): los resultados de las escalas SEAMS y BMQ fueron comparados con la evaluación del médico tratante durante la consulta médica, quien realizó preguntas directas al paciente acerca de la toma de los medicamentos y la ATF, junto con la evaluación global de actividad de la enfermedad (con parámetros clínicos y bioquímicos), en la que se considera que la falta de apego predispone a mayor actividad de la Ell y recaídas, ${ }^{24}$ calificando la adherencia por medio de la EVA, la cual fue considerada como estándar de oro. La EVA va de 0 a 10, donde los pacientes fueron clasificados como adherentes cuando el médico tratante calificaba con una puntuación de 9 o 10 y como no adherentes, cuando las puntuaciones eran $\leq 8 .{ }^{25}$

\section{Análisis estadístico}

La descripción de las características demográficas y clínicas se realizó con frecuencias y porcentajes para las variables categóricas y medias y desviaciones estándar (DE) para las variables continuas.

Para probar las propiedades clinimétricas de la SEAMS se siguieron los siguientes procedimientos:

- Se elaboró una matriz de correlación de los reactivos; aquellos con un coeficiente menor a 0.20 serían eliminados de análisis posteriores.

- Se realizó un análisis factorial exploratorio con rotación varimax para probar la validez de constructo; los reactivos con comunalidades menores a 0.40 fueron excluidos.

- Se determinó la consistencia interna mediante el coeficiente alfa de Cronbach.

- Se realizó una curva ROC (recevier operating characteristic) para determinar la puntuación más adecuada de la SEAMS en términos de sensibilidad y especificidad.

Para el BMQ:

- Se empleó el coeficiente kappa de Cohen para determinar la concordancia entre el reporte de adherencia del instrumento y la clasificación realizada por los médicos tratantes.

- Se definió la capacidad predictiva de las cuatro dimensiones del $B M Q$, con distintos niveles de adherencia de acuerdo con la valoración clínica.

\section{Resultados}

Se incluyeron 149 pacientes, de los cuales $50.3 \%$ $(n=75)$ era del sexo femenino. La edad promedio de los pacientes fue de 44.2 años (DE = 13.6, rango $=20-78)$. La mayoría de los pacientes incluidos tenía diagnóstico de $\mathrm{CUCl}(81.2 \%, \mathrm{n}=121)$ y $18.8 \%$ ( $n=28$ ) de enfermedad de Crohn. Todos los pacientes se encontraban bajo tratamiento farmacológico al momento de su inclusión en el estudio (mesalazina $77.9 \%, n=116$; azatioprina $36.9 \%, n=55$; esteroides $33.6 \%$ y sulfasalazina $8.7 \%, n=13$, principalmente). De acuerdo con la EVA, la puntuación promedio de adherencia asignada por los médicos tratantes fue de $7.8(\mathrm{DE}=1.4,4-10) ; 45.6 \%(n=68)$ de los pacientes fueron clasificados como adherentes y $54.4 \%(n=81)$ como no adherentes.

\section{SEAMS, propiedades clinimétricas}

Los rangos de correlación de los 13 reactivos de la SEAMS se encontraron entre 0.58 y 0.80 , por lo que ningún reactivo fue eliminado y todos fueron incluidos en el análisis. Los resultados mostraron que un solo 
factor (Tabla 1) explicó $54.1 \%$ de la varianza, modelo adecuado, de acuerdo con un valor Kaiser-MeyerOlkin de 0.91 y una prueba de esfericidad de Bartlett altamente significativa $(p<0.001)$. Este factor es altamente confiable (alfa de Cronbach $=0.92$ ).

Al evaluar el mejor punto de corte de la SEAMS para identificar a los pacientes adherentes se encontró que la puntuación de 33 mostraba valores de sensibilidad y especificidad más estables, con un valor del área bajo la curva de 0.92 (Figura 1), indicativo de adecuada capacidad discriminante de SEAMS.

\section{$B M Q$, propiedades clinimétricas}

En la primera dimensión de evaluación del $B M Q$, adherencia, $45.6 \%(n=68)$ de los pacientes fue clasificado como adherente. En las siguientes dimensiones, enfocadas a la evaluación de las barreras para la adherencia, se observó que $28.9 \%(n=43)$ reportó creencias negativas hacia los medicamentos, $47.7 \%$ $(n=71)$ barreras de recuerdo en la toma de los medicamentos y $51 \%(n=76)$, barrera de acceso a los medicamentos. El coeficiente kappa de Cohen del $\mathrm{BMQ}$ en la valoración de la adherencia, en contraste con el reporte de los médicos tratantes fue de 1.0 $(p<0.001)$, indicativo de concordancia ideal.

En la Tabla 2 se detallan los valores de sensibilidad, especificidad y predictivos de las dimensiones del $\mathrm{BMQ}$ para identificar a los pacientes con adecuada
Tabla 1. Análisis factorial de la Self-Efficacy for Appropriate Medication Use Scale (SEAMS) en pacientes mexicanos con enfermedad inflamatoria intestinal

\begin{tabular}{|c|c|}
\hline Reactivo & Factor 1 \\
\hline $\begin{array}{l}\text { 1. Cuando usted toma varios medicamentos diferentes } \\
\text { cada día. }\end{array}$ & 0.669 \\
\hline $\begin{array}{l}\text { 2. Cuando usted toma medicamentos más de una vez al } \\
\text { día. }\end{array}$ & 0.774 \\
\hline 3. Cuando usted está lejos de casa. & 0.819 \\
\hline 4. Cuando usted tiene planeado un día ocupado & 0.846 \\
\hline 5. Cuando le causan algunos efectos secundarios. & 0.784 \\
\hline 6. Cuando nadie le recuerda tomar su medicamento. & 0.761 \\
\hline $\begin{array}{l}\text { 7. Cuando el horario para tomar el medicamento no es } \\
\text { conveniente. }\end{array}$ & 0.690 \\
\hline 8. Cuando su rutina normal se estropea. & 0.661 \\
\hline $\begin{array}{l}\text { 9. Cuando usted no está seguro de cómo tomar el } \\
\text { medicamento. }\end{array}$ & 0.730 \\
\hline $\begin{array}{l}\text { 10. Cuando usted no está seguro en qué horario del día } \\
\text { debe tomar el medicamento. }\end{array}$ & 0.732 \\
\hline $\begin{array}{l}\text { 11. Cuando usted se siente enfermo (como tener un } \\
\text { resfriado o gripe). }\end{array}$ & 0.720 \\
\hline $\begin{array}{l}\text { 12. Cuando usted consigue un repuesto de sus } \\
\text { medicamentos viejos y algunas de las píldoras se ven } \\
\text { diferentes. }\end{array}$ & 0.637 \\
\hline 13. Cuando su médico le cambia sus medicamentos. & 0.712 \\
\hline Valor eigen & 7.03 \\
\hline Varianza & 54.14 \\
\hline
\end{tabular}

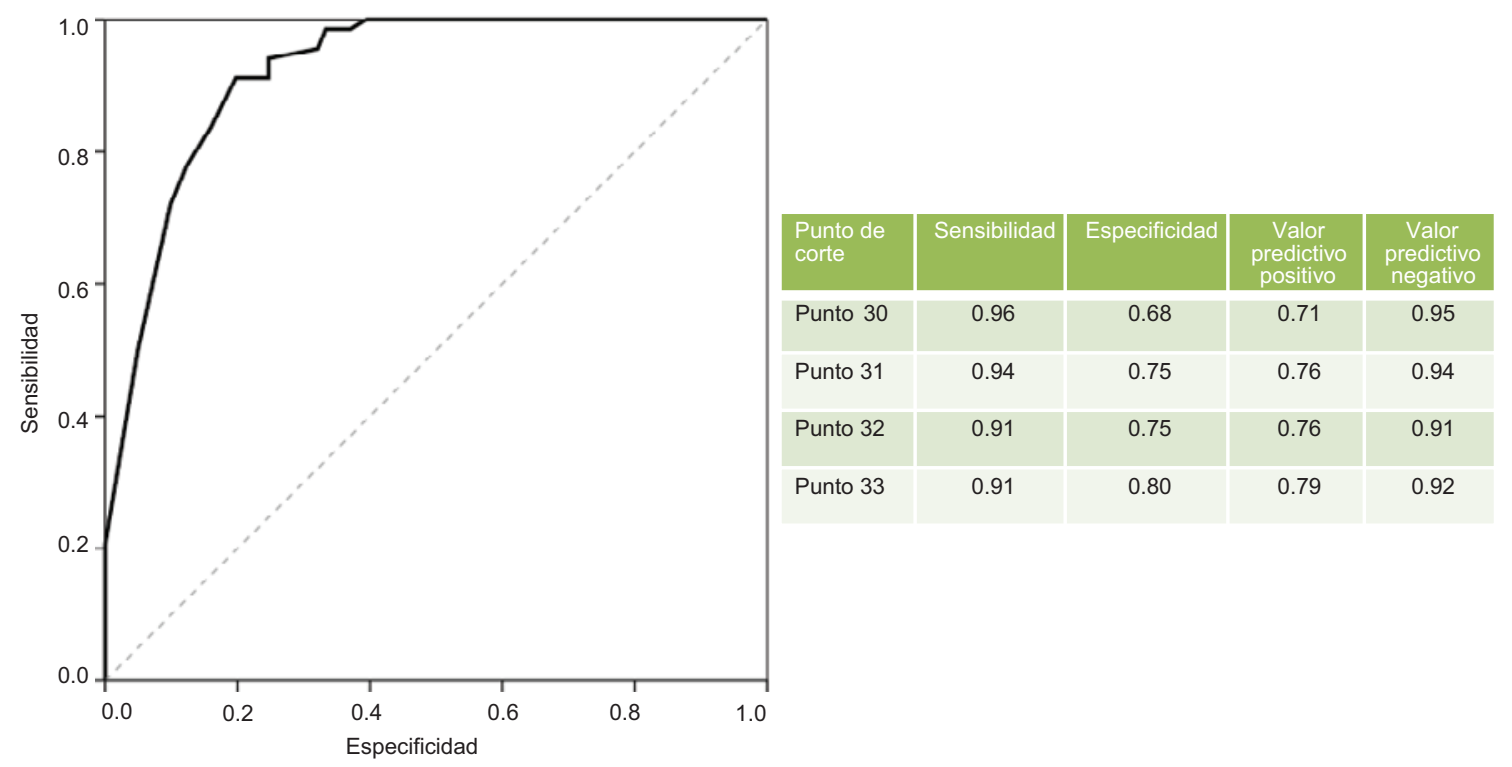

Figura 1. Puntos de corte de la escala SEAMS. Sensibilidad, especificidad y valores predictivos. 
Tabla 2. Capacidad predictiva de las dimensiones del Belief Medicines Questionnaire (BMQ)

\begin{tabular}{|c|c|c|c|c|}
\hline EVA & Sensibilidad & Especificidad & Valor predictivo positivo & Valor predictivo negativo \\
\hline \multicolumn{5}{|c|}{ BMQ-Adherencia } \\
\hline Adherencia 7 & 1.00 & 0.36 & 0.57 & 1.00 \\
\hline Adherencia 8 & 1.00 & 0.60 & 0.68 & 1.00 \\
\hline Adherencia 9 & 1.00 & 1.00 & 1.00 & 1.00 \\
\hline \multicolumn{5}{|c|}{ BMQ-Creencias negativas hacia los medicamentos } \\
\hline Adherencia 7 & 0.90 & 0.42 & 0.79 & 0.62 \\
\hline Adherencia 8 & 0.82 & 0.70 & 0.87 & 0.61 \\
\hline Adherencia 9 & 0.58 & 0.84 & 0.90 & 0.44 \\
\hline \multicolumn{5}{|c|}{ BMQ-Barrera de recuerdo } \\
\hline Adherencia 7 & 0.97 & 0.35 & 0.58 & 0.93 \\
\hline Adherencia 8 & 0.90 & 0.54 & 0.64 & 0.86 \\
\hline Adherencia 9 & 0.86 & 0.91 & 0.90 & 0.88 \\
\hline \multicolumn{5}{|c|}{ BMQ-Barrera de acceso a los medicamentos } \\
\hline Adherencia 7 & 0.75 & 0.14 & 0.48 & 0.34 \\
\hline Adherencia 8 & 0.64 & 0.30 & 0.49 & 0.45 \\
\hline Adherencia 9 & 0.47 & 0.56 & 0.53 & 0.51 \\
\hline
\end{tabular}

EVA = escala visual análogo, EVA 9=adherencia adecuada, EVA 7-8 = adherencia moderada.

adherencia de acuerdo con el reporte del médico tratante (EVA $\geq 9)$ y aquellos con adherencia moderada $(E V A=7-8)$. La dimensión de adherencia del BMQ tuvo valores ideales para la identificación de adecuada adherencia, si bien su especificidad y valor predictivo positivo disminuyeron para identificar a los pacientes con adherencia moderada. Por su parte, las dimensiones de creencias negativas hacia los medicamentos y barrera de acceso a los medicamentos indicaron valores inadecuados para la predicción de adecuada y moderada adherencia. La dimensión barrera de recuerdo tuvo adecuados valores para identificar a los pacientes con adecuada adherencia, a diferencia de aquellos con adherencia moderada, cuyos valores de especificidad y predictivo positivo fueron bajos.

\section{Discusión}

La inadecuada ATF impacta negativamente en el curso clínico de diversas enfermedades crónicas, especialmente en la Ell, en la que es indispensable tomar fármacos de por vida para mantener la remisión. Se ha descrito que la falta de ATF conlleva desenlaces clínicos fatales, así como mayores ingresos hospitalarios, los cuales impactan negativamente en la calidad de vida de los pacientes. ${ }^{4}$
El estudio que se presenta es el primero en México acerca de la validación y traducción de dos instrumentos útiles para vigilar la ATF en pacientes con Ell. De acuerdo con nuestros resultados, el análisis factorial del SEAMS traducido indicó que todo el instrumento apuntaba a la dimensión autoeficacia, a diferencia de la versión original, ${ }^{15}$ donde el análisis factorial reveló dos dimensiones de la autoeficacia de los medicamentos: la autoeficacia para tomar medicamentos en circunstancias difíciles, como cuando los pacientes están ocupados, lejos de casa o tienen que tomar múltiples medicamento, y la autoeficacia para tomar medicamentos en circunstancias inciertas o cambiantes, como cuando el paciente no está seguro acerca de cómo tomarlos. Resultados similares obtuvieron otros grupos que tradujeron y validaron los instrumentos. Por su parte, Pedrosa et al. ${ }^{26}$ validaron el SEAMS en una población brasileña, en la que el análisis discriminó para dos factores al igual que la versión original.

El mejor punto de corte en la escala SEAMS para discriminar pacientes adherentes fue 33 puntos, a partir del cual el área bajo la curva fue de 0.92 (sensibilidad de $91 \%$ y especificidad de $80 \%$ ), lo que indicó adecuada capacidad discriminante del instrumento. En China, Dong et al. ${ }^{27}$ validaron y tradujeron la escala SEAMS 
al idioma chino en pacientes con infarto cerebral; el mejor de corte fue en 32 puntos, similar al nuestro.

Por otra parte, de acuerdo con nuestro análisis factorial de la escala $\mathrm{BMQ}$, únicamente dos de las cuatro dimensiones del instrumento fueron predictoras de adherencia en pacientes con Ell: adherencia en general y barreras para el recuerdo (sensibilidad de $86 \%$ y especificidad de $91 \%$ ); las dimensiones creencias negativas hacia los medicamentos y barrera de acceso a los medicamentos mostraron valores inadecuados para la predicción de adecuada adherencia.

En el estudio ALIGN, ${ }^{28}$ para medir la ATF se utilizó la escala $B M Q$, con la cual se describió que una proporción significativa de pacientes (52.4\%) presentaba creencias negativas en relación con la medicación y, sin embargo, mostraba adhesión al tratamiento a pesar de esta barrera, hallazgos similares a los nuestros. Con el cuestionario BMQ para medir ATF, Castro et al. ${ }^{29}$ encontraron que la dimensión de baja adherencia se asoció con altos niveles de preocupación acerca de la medicación empleada para el control de la Ell, mientras que las puntuaciones elevadas respecto al potencial daño de los medicamentos en general se asociaron signicativamente con falta de adherencia.

Entre las limitaciones de este estudio se identifica que la validación de los instrumentos únicamente se realizó en un centro de referencia nacional, en un hospital de tercer nivel, lo que impide la generalización de los resultados a toda la población mexicana con diagnóstico de Ell. A pesar de ello, BMQ y SEAMS validados en población mexicana con Ell brindan información clave de la ATF que permitirá crear estrategias que optimicen el apego terapéutico de los pacientes, con la consecuente mejoría de los desenlaces clínicos.

\section{Conclusiones}

La traducción al español y la validación de las escalas SEAMS y BMQ mostraron propiedades clinimétricas adecuadas, indicativas de fiabilidad para medir ATF en pacientes mexicanos con Ell.

\section{Bibliografía}

1. Abraham C, Cho JH. Inflammatory bowel disease. N Engl J Med. 2009; 361:2066-2078.

2. Sabaté E. Adherence to long-term therapies: evidence for action. Suiza: World Health Organization; 2003.

3. Mårdby AC, Åkerlind I, Jörgensen T. Beliefs about medicines and self-reported adherence among pharmacy clients. Patient Educ Couns. 2007; 69:158-164.

4. Kane S V, Robinson A. Understanding adherence to medication in ulcerative colitis-innovative thinking and evolving concepts. Aliment Pharmacol Ther. 2010;32:1051-1058.
5. Mitra D, Hodgkins $P$, Yen L, Davis KL, Cohen RD. Association between oral 5-ASA adherence and health care utilization and costs among patients with active ulcerative colitis. BMC Gastroenterol. 2012;12:132.

6. Kane SV, Chao J, Mulani PM. Adherence to infliximab maintenance therapy and health care utilization and costs by Crohn's disease patients. Adv Ther. 2009;26:936-946.

7. Higgins PD, Rubin DT, Kaulback K, Schoenfield PS, Kane SV. Systematic review: impact of non-adherence to 5 -aminosalicylic acid products on the frequency and cost of ulcerative colitis flares. Aliment Pharmacol Ther. 2009;29:247-257.

8. Van Staa TP, Card T, Logan RF, Leufkens HG. 5-aminosalicylate use and colorectal cancer risk in inflammatory bowel disease: a large epidemiological study. Gut. 2005;54:1573-1578.

9. Khan N, Abbas AM, Bazzano LA, Koleva YN, Krousel-Wood M. Longterm oral mesalazine adherence and the risk of disease flare in ulcerative colitis: nationwide 10-year retrospective cohort from the veterans affairs healthcare system. Aliment Pharmacol Ther. 2012;36:755-764.

10. Osterberg L, Blaschke T. Adherence to medication. N Engl J Med. 2005;353:487-497.

11. Nguyen TMU, La-Caze A, Cottrell N. What are validated self-report adherence scales really measuring? A systematic review. Br J Clin Pharmacol. 2014;77:427-445.

12. Morisky DE, Green LW, Levine DM. Concurrent and predictive validity of a self-reported measure of medication adherence. Med Care. 1986: 24:67-74.

13. Horne R, Weinman J. Self-regulation and self-management in asthma: exploring the role of illness perceptions and treatment beliefs in explaining non-adherence to preventer medication. Psychol Heal. 2002;17:17-32.

14. Horne R, Weinman J, Hankins M. The beliefs about medicines questionnaire: the development and evaluation of a new method for assessing the cognitive representation of medication. Psychol Heal. 1999;14:1-24.

15. Risser J, Jacobson TA, Kripalani S. Development and psychometric evaluation of the self-efficacy for Appropriate Medication Use Scale (SEAMS) in low-literacy patients with chronic disease. 2007;15:203-219.

16. Bandura A. Self-efficacy mechanism in human agency. Am Psychol. 1982;37:122.

17. Burke LE, Dunbar-Jacob JM, Hill MN. Compliance with cardiovascular disease prevention strategies: a review of the research. Ann Behav Med. 1997;19:239-263.

18. Perpiñá-Tordera M, Martínez-Moragón EM, Belloch-Fuster A, LlorisBayo A, Pellicer-Císcar C. Spanish asthma patients' beliefs about health and medicines: validation of 2 questionnaires. Arch Bronconeumol. 2009; 45:218-223.

19. Fall E, Gauchet A, Izaute M, Horne R, Chakroun N. Validation of the French version of the Beliefs about Medicines Questionnaire (BMQ) among diabetes and HIV patients. Rev Eur Psychol Appliquée/European Rev Appl Psychol. 2014;64:335-343.

20. Jónsdóttir H, Friis S, Horne R, Pettersen KI, Reikvam Å, Andreassen OA. Beliefs about medications: measurement and relationship to adherence in patients with severe mental disorders. Acta Psychiatr Scand. 2009; 119:78-84.

21. Neame R, Hammond A. Beliefs about medications: a questionnaire survey of people with rheumatoid arthritis. Rheumatology (Oxford). 2005; 44:762-767.

22. Horne R, Parham R, Driscoll R, Robinson A. Patient's attitudes to medicines and adherence to maintenance treatment in inflammatory bowel disease. Inflamm Bowel Dis. 2009;15:837-844.

23. Pan $Y$, De la Puente M. Census bureau guideline for the translation of data collection instruments and supporting materials: documentation on how the guideline was developed. Surv Methodol. 2005;6.

24. Bluett J, Morgan C, Thurston L, Plant D, Hyrich KL, Morgan AW, et al. Impact of inadequate adherence on response to subcutaneously administered anti-tumour necrosis factor drugs: results from the Biologics in Rheumatoid Arthritis Genetics and Genomics Study Syndicate cohort. Rheumatology (Oxford). 2014;54:494-499.

25. Giordano TP, Guzmán D, Clark R, Charlebois ED, Bangsberg DR. Measuring adherence to antiretroviral therapy in a diverse population using a visual analogue scale. HIV Clin Trials. 2004;5:74-79.

26. Pedrosa RB, Rodrigues RC, Oliveira HC, Alexandre NM. Construct validity of the Brazilian version of the Self-Efficacy for Appropriate Medication Adherence Scale. J Nurs Meas. 2016;24:18-31.

27. Dong XF, Liu YJ, Wang AX, Lv PH. Psychometric properties of the Chinese version of the Self-Efficacy for Appropriate Medication Use Scale in patients with stroke. Patient Prefer Adherence. 2016;10:321-327.

28. Michetti P, Weinman J, Mrowietz U, Smolen J, Louis E, Peyrin-Biroulet L, et al. Impact of treatment-related beliefs on medication adherence in immune-mediated inflammatory diseases: results of the global ALIGN study. Adv Ther. 2017;34:91-108.

29. De Castro ML, Sanromán L, Martín A, Martínez N, Hernández V, Del Campo V, et al. Valoración de la adhesión terapéutica en la enfermedad inflamatoria intestinal. Comparación entre una escala de autoevaluación y un índice farmacéutico de dispensación de medicamentos. Rev Esp Enferm Dig. 2017;109:542-551. 